\title{
ROCK inhibitor Y-27632 protects rats against cerebral ischemia/reperfusion-induced behavioral deficits and hippocampal damage
}

\author{
LIHE LI and BAOYANG LIU \\ Department of Clinical Laboratory, Baodi District People's Hospital, Tianjin 301800, P.R. China
}

Received November 9, 2018; Accepted June 24, 2019

DOI: $10.3892 / \mathrm{mmr} .2019 .10584$

\begin{abstract}
Cerebral ischemic injury is a major cause of death and long-term disability worldwide that leads to neurological and behavioral deficits, and for which successful treatments are still lacking. Ras homolog family member A (RhoA) and Rho-associated coiled-coil containing protein kinase (ROCK) are associated with the growth of neurons and the movement of neuronal growth cones. RhoA/ROCK inhibitors have been demonstrated to promote the recovery of motor function following nerve injury, but the underlying mechanism requires further investigation. The present study aimed to investigate the effects of the ROCK inhibitor Y-27632 on middle cerebral artery occlusion (MCAO)-induced cerebral ischemic injury. Rats were randomly assigned to the Control, Y-27632, MCAO + Vehicle or MCAO + Y-27632 group. Firstly, infarct volume, cognitive ability and cerebral injury were assessed. Secondly, indicators of cerebral inflammation, oxidative stress and apoptosis were evaluated. Finally, the expression of recombinant glial fibrillary acidic protein (GFAP) and allograft inflammatory factor 1 (AIF1) in the brain were measured to assess the activation of astrocytes and microglia, respectively. The results showed that Y-27632 effectively increased the survival rate and behavioral performance of rats, and attenuated the cerebral injury, oxidative stress and cerebral inflammation levels following MCAO. The disturbance in hippocampal neurons caused by MCAO was also alleviated following treatment with Y-27632. Neuronal apoptosis was also decreased following Y-27632 treatment, as demonstrated by the TUNEL assay and the expression levels of Caspases-3, 8 and 9 and $\mathrm{Bax} / \mathrm{Bcl}-2$ ratio. The levels of GFAP and AIF1 were increased by MCAO and further promoted by Y-27632, indicating the activation of astrocytes and microglia. In conclusion,
\end{abstract}

Correspondence to: Dr Lihe Li, Department of Clinical Laboratory, Baodi District People's Hospital, 8 Guangchuan Road, Baodi, Tianjin 301800, P.R. China

E-mail: lihe.tianjin@outlook.com

Key words: Rho associated coiled-coil containing protein kinase, Y-27632, cerebral ischemia/reperfusion, oxidative stress, inflammation, apoptosis the present study offered evidence of a protective effect of Y-27632 administration on cerebral ischemia/reperfusion induced behavioral and hippocampal damage by activating astrocytes and microglia.

\section{Introduction}

Cerebrovascular disease is becoming a prominent public health concern. Due to its high rates of disability, morbidity and mortality, cerebrovascular disease not only has a strong impact on a patient's quality of life, but is also accompanied by heavy economic burdens for the patients' families and society (1). Currently, treatment of cerebrovascular disease consists in the surgical removal of blocked blood vessels in order to improve the blood supply in and around the lesion site (2). Once the blood supply improves, the infarcted area is prone to develop severe cerebral ischemia/reperfusion (CIR) injury. There are several pathological mechanisms involved in the process of CIR damage, including glutamate-induced excitotoxicity, loss of ionic homeostasis, energy failure, inflammatory response, increased oxidative stress and apoptosis (3). The accumulation of reactive oxygen species (ROS) in cells can directly lead to cell necrosis and indirectly induce cell senescence, apoptosis and necrosis by activating oxidative signaling pathways (4). Local excessive inflammatory reactions after cerebral ischemia-reperfusion also cause tissue damage. In recent years, calcium ion antagonist, radical scavengers and other neuroprotective drugs have been developed and used for the treatment of CIR injury (5). However, the possible side effects of these treatments, including drug resistance, cerebral hemorrhage and gastrointestinal irritation, may exceed the clinical benefits of long-term therapy.

Ras homolog family member A (RhoA) has GTPase activity and acts as a signal transducer or as a molecular switch in cell signaling pathways. It may also act on the cytoskeleton and regulates the response of actin to extracellular signals. Rho associated coiled-coil containing protein kinase (ROCK) can catalyze the conversion of inactive RhoA to active RhoA (6). In neurons, activation of RhoA leads to axonal retraction; conversely, inactivation of RhoA or ROCK promotes the growth of neurons and the movement of neuron growth cones. Therefore, RhoA/ROCK pathway is closely associated with the growth of neuron and their axons (7). Y-27632 is a type of synthetic pyridine complex that can be introduced in cells via 
vectors. Y-27632 is a cell-permeable, highly potent and selective inhibitor of Rho-associated, coiled-coil containing protein kinase (ROCK). It binds intracellularly to the catalytic site of the upstream effector of the Rho protein ROCK (both ROCK1 and 2), thereby inhibiting its kinase activity. Y-27632 inhibits both ROCK1 $(\mathrm{Ki}=220 \mathrm{nM})$ and $\operatorname{ROCK} 2(\mathrm{Ki}=300 \mathrm{nM})$ by competing with ATP for binding to the catalytic site. Y-27632 has been shown to inhibit ROCK kinase activity in epithelial cells, endothelial cells, smooth muscle cells and neurons (8). It has also been found that Y-27632 promotes neurite outgrowth in PC12 cells (9). Y-27632 has also been used in treatments following spinal cord injury, for it is known to promote neurite outgrowth and axonal regeneration in neurons (10). However, the effect of Y-27632 on CIR injury needs to be studied. IR injury is also known to stimulate the activation and proliferation of astrocytes and microglia to protect brain tissues $(11,12)$. Glial fibrillary acidic protein (GFAP), found in astrocytes and ependymal cells, is a type of cellular microfilament with multiple functions in cell activity, and its expression may be affected following CIR.

Therefore, the present study, aimed to investigate the effects of the ROCK inhibitor Y-27632 on CIR injury and to explore its underlying mechanism of action. The cognitive ability, cerebral infarct, inflammation, oxidative stress and apoptosis in rats with middle cerebral artery occlusion (MCAO) were used to evaluate the effect of Y-27632 on CIR. The levels GFAP and allograft inflammatory factor 1 (AIF1) in neurons were assessed by western blotting to evaluate the activation of astrocytes and microglia in the context of CIR.

\section{Materials and methods}

Animals. A total of 310 male Sprague-Dawley rats, weighing 230-250 g, were purchased from Tianjin Medical University animal center. Animals were housed at $25^{\circ} \mathrm{C}$ with a $12-\mathrm{h}$ day/night cycle in the Animal Center of Baodi District People's Hospital with food and water available ad libitum. The procedures of the present study followed international guidelines of animal care (NIH publication No. 92-3415, revised 1999) and were approved by the Animal Care Committee in Baodi District People's Hospital.

Experimental protocol. The experimental protocol is summarized in Fig. 1. For biochemical evaluation, hematoxylin and eosin (H\&E) staining, western blotting and behavioral assessment, 80 rats were randomly grouped into Control, $\mathrm{Y}-27632, \mathrm{MCAO}+$ Vehicle and MCAO + Y-27632 groups, with 20 animals in each group. Rats in the Control group were operated on like the experimental MCAO ones, but the nylon filament was not introduced to the carotid artery. Rats in the Y-27632 group received Y-27632 injection and the same operation as Control. Rats in MCAO + Vehicle group received MCAO surgery and were injected with saline 120 min later. Rats in MCAO + Y-27632 group received MCAO surgery and were injected with Y-27632 120 min later. After MCAO, animals were allowed to rest for $24 \mathrm{~h}$. Then, half the animals in each group were sacrificed and used in for biochemical, $\mathrm{H} \& \mathrm{E}$ and western blot analyses, while the remaining half was used for neurological deficit scoring. After $48 \mathrm{~h}$, these animals were tested in the water maze. For survival rate studies, 64 rats were randomly grouped into the Control group $(n=12), Y-27632$ group $(n=12), M C A O+$ Vehicle group $(\mathrm{n}=20)$ and MCAO + Y-27632 group $(n=20)$. Survival checks were performed at 24, $48 \mathrm{~h}$ and 7 days after MCAO surgery. The animal health and behavior were monitored twice a day during the experiments. Survival checks were performed at 1 , 2 and 7 days following MCAO surgery; the inability of rats to right themselves in $30 \mathrm{sec}$ after being placed on their side was considered a humane endpoint and were euthanized by overdose of sodium pentobarbital $(150 \mathrm{mg} / \mathrm{kg})$. All Rats were euthanatized with intraperitoneal injection of an overdose of sodium pentobarbital $(150 \mathrm{mg} / \mathrm{kg})$ at the end of the study. Death was verified by checking the heartbeat. Rats would be euthanatized if serious infection occurred and remained uncontrollable. However, no rat was euthanized prior to the end of the study due to ill health.

MCAO procedure. The MCAO procedure was similar to that of Yang et al (13). Briefly, rats were anesthetized with ketamine (100 mg/kg, intra-muscularly) and xylazine (7.5 mg/kg, intra-muscularly) and mounted on a wooden plate. A $3 \mathrm{~cm}$ long incision was made in the center of neck to expose the common carotid and external carotid arteries. After these two arteries were ligated, a nylon filament (diameter, $0.25 \mathrm{~mm}$ ) was inserted into the internal carotid artery and advanced for $20 \mathrm{~mm}$. MCAO lasted for $60 \mathrm{~min}$ to generate ischemia, and the nylon filament was pulled out to allow reperfusion of the injured site. The body temperature of animals was kept at $37 \pm 1^{\circ} \mathrm{C}$ with a heating blanket during the IR process.

Intracerebroventricular injection. After $30 \mathrm{~min}$ since reperfusion, rats were anesthetized with ketamine $(100 \mathrm{mg} / \mathrm{kg}$, intra-muscularly) and xylazine ( $7.5 \mathrm{mg} / \mathrm{kg}$, intra-muscularly). After the head of each rat was mounted in a stereotaxic instrument, the skull was revealed by a midline incision. A craniotomy was drilled in the right lateral cerebral ventricle at the following stereotaxic coordinates relative to the bregma: $1.00 \mathrm{~mm}$ anteroposterior, $1.5 \mathrm{~mm}$ mediolateral and $2.00 \mathrm{~mm}$ dorsoventral. In the MCAO + Vehicle group, $10 \mu \mathrm{l}$ artificial cerebrospinal fluid was injected into the right lateral cerebral ventricle with a microinjector. In the Y-27632 and

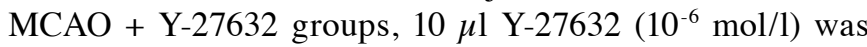
injected in the same manner. Afterwards, the incision was closed with a wound clip. Ampicillin $(20 \mathrm{mg} / \mathrm{kg}$, twice per day) and meloxicam $(0.2 \mathrm{mg} / \mathrm{kg}$, once per day) were injected intra-muscularly to prevent wound infection and pain.

\section{2,3,5-Triphenyltetrazolium chloride (TTC) staining.} Following experiments, the brains of animals were collected for histological analysis. After $20 \mathrm{~min}$ freezing at $-20^{\circ} \mathrm{C}, 5$ to 6-1.7 mm thick coronal sections were obtained and placed in $2 \%$ TTC phosphate buffer solution (23.9 mM TTC in $0.05 \mathrm{M}$ phosphate buffer solution, $\mathrm{pH}$ 7.5). These sections were stained at $37^{\circ} \mathrm{C}$ for $30 \mathrm{~min}$ in the dark, and then images were capture with a digital camera. The normal tissue was stained red, while the infarcted tissue exhibited white coloration. The picture analyzed using a pathological image analysis computer system, and the infarct size of each layer was measured to calculate the infarct volume. 


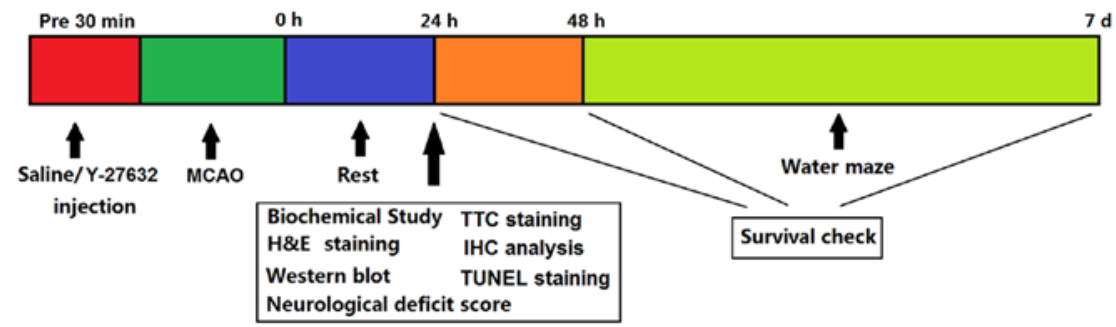

Figure 1. Experimental protocol. A total of $120 \mathrm{~min}$ after MCAO, rats were injected with saline or Y-27632. Rats were allowed to rest for 24 h after MCAO, then half of the animals were sacrificed and used for biochemical, hematoxylin and eosin, 2,3,5-triphenyltetrazolium chloride, immunohistochemical, terminal deoxynucleotidyl transferase dUTP nick end labeling and western blot analyses. The remaining rats were used for neurological deficit scoring. After $48 \mathrm{~h}$, these animals were tested in a water maze study. Survival checks were performed at 24, $48 \mathrm{~h}$ and 7 days after MCAO surgery. MCAO, middle cerebral artery occlusion.

Neurological deficit scoring. Neurological deficit scoring was performed as previously described (14). At $48 \mathrm{~h}$ after MCAO, the potential deficit points of animals were evaluated as follows: no nerve injury symptom, 0 points; contralateral papillary flexion, 1 point; reduced contralateral forepaw clenched grip, 2 points; spontaneous movement in all directions and circle to the opposite side when pulling their tails, 3 points; loss of consciousness, 4 points.

Water maze study. The water maze study was performed at $48 \mathrm{~h}$ after MCAO following the methodology described by Ahmadi et al (15). Briefly, a black circular tank (120 cm diameter, $80 \mathrm{~cm}$ high) was divided into four equal quadrants and used as the water maze. The depth and the temperature of water were kept at $30 \mathrm{~cm}$ and $25 \pm 1^{\circ} \mathrm{C}$, respectively. A circular platform was placed $1.5 \mathrm{~cm}$ under the water in a quadrant. A video camera was used to track the travel path and duration of rats. Beginning at $48 \mathrm{~h}$ after MCAO, the animals were trained for five consecutive days with the platform located in the same position. The escape latency, traveled length and mean speed of animals were calculated from the recorded video. There was no significant difference between groups in the mean speed, indicating that physical deficit was not caused by the MCAO procedure. On the sixth day, the platform was removed. The rats were allowed to swim in the water maze for $60 \mathrm{~s}$, and the number of times they crossed the platform location (crosses) and the duration they spent in the target quadrant were recorded by the video camera.

Measurement of oxidative and pro-inflammatory factors in the brain tissue. At $24 \mathrm{~h}$ after MCAO, animals were sacrificed and brains were harvested and sliced into $1.0 \mathrm{~mm}$ thick slices. A total $100 \mathrm{mg}$ brain tissue of the infarcted area was collected and homogenized in PBS (10 mM, pH=7.4). Next, they were centrifuged at $12,000 \mathrm{x}$ g at $4^{\circ} \mathrm{C}$ for $30 \mathrm{~min}$ before the supernatant was collected. The levels of oxidative markers [malondialdehyde (MDA), (8-hydroxy-2-deoxyguanosine (8-OHdG), 3-nitrotyrosine (3-NT)] and pro-inflammatory factors [tumor necrosis factor (TNF)- $\alpha$, interleukin (IL)-1 $\beta$, IL-6] in the supernatant were measured using corresponding assay kits (Sigma-Aldrich; Merck KGaA), following the manufacturer's protocol. Their concentration was calculated with absorbance values which were detected by a microplate reader (Azure Biosystems, Inc.). The protein levels were measured with a protein assay kit (Nanjing Jiancheng Bioengineering Institute) using the bicinchoninic acid method. All the concentrations were normalized to the protein level.
Hematoxylin and eosin $(H \& E)$ staining. A total of $24 \mathrm{~h}$ following $\mathrm{MCAO}$, rats were anesthetized with ketamine $(100 \mathrm{mg} / \mathrm{kg}$, intra-muscularly) and xylazine (7.5 mg/kg, intra-muscularly) and perfused with cold saline containing heparin $(40 \mathrm{mg} / \mathrm{l})$ and $4 \%$ paraformaldehyde. After the rats were fixed on a wooden plate, the needle used for perfusion was inserted into the aortic root of the rats from the left apex. After the needle was fixed, the perfusion with cold saline containing heparin started. When no blood was seen in the perfusion solution, which indicated that the blood in the animal was completely replaced by cold saline containing heparin, the perfusion solution was changed to $4 \%$ paraformaldehyde. $1 \mathrm{~h}$ later, the brains were removed, fixed in $4 \%$ paraformaldehyde and embedded in paraffin at room temperature. Coronal sections ( $6 \mu \mathrm{m}$ thick) were taken every millimeter from -1.3 to $-6.3 \mathrm{~mm}$ relative to the bregma. Hippocampal sections of the ipsilateral side of the MCAO from eight rats in each group were collected and stained with $H \& E$ at room temperature for $5 \mathrm{~min}$ to analyze the neuronal injury. The numbers of nucleoli and cytoplasmic Nissl bodies and pyknosis-positive neurons were compared within groups. A VS120 Virtual Slide Microscope (Olympus Corporation) and cellSens Imaging Software (version 2.2; Olympus Corporation) were used to capture and analyze the images.

Immunohistochemical (IHC) analysis. IHC analysis of neuron specific enolase (NSE), a marker of cerebral damage, was performed to evaluate CIR. Hippocampal sections of the ipsilateral side of the MCAO from eight rats in each group were collected. Dewaxing and hydration were first carried out with xylene and alcohol. Endogenous peroxidase activity was then blocked by the addition of $0.3 \%$ hydrogen peroxide at room temperature for $30 \mathrm{~min}$, and antigen retrieval was performed using $0.01 \mathrm{M}$ citrate buffer. Next, non-specific antibodies were blocked by incubation with $5 \%$ goat serum (Beyotime Institute of Biotechnology) at $37^{\circ} \mathrm{C}$ for $45 \mathrm{~min}$. They were then incubated with primary antibody against NSE (cat. no. SAB4500768; 1:1,000; Sigma-Aldrich; Merck KGaA) at $4{ }^{\circ} \mathrm{C}$ overnight and with the goat anti-rabbit IgG secondary antibody conjugated to biotin (cat. no. SAB4600006, 1:5,000, Sigma-Aldrich; Merck $\mathrm{KGaA}$ ) at $37^{\circ} \mathrm{C}$ for $1 \mathrm{~h}$. Afterwards, the sections were washed with PBS three times and incubated with streptavidin conjugated to horseradish peroxidase (cat. no. 18-152, $10 \mu \mathrm{g} / \mathrm{ml}$, Sigma-Aldrich; Merck KGaA) at room temperature for $1 \mathrm{~h}$. The color was developed using DAB coloring solution, then counterstained with hematoxylin at 
room temperature for $10 \mathrm{~min}$. Finally, the slices were dehydrated and sealed with xylene and alcohol. Representative images were taken from the hippocampal region in a total of four different sections per animal using a VS120 Virtual Slide Microscope (Olympus Corporation) at a magnification of $x 200$. The number of immunoreactive signals of NSE was counted in four randomly selected fields per section.

Terminal deoxynucleotidyl transferase dUTP nick end labeling (TUNEL) staining. Hippocampal sections of the ipsilateral side of the MCAO from eight rats in each group were collected. Dewaxing and hydration were first carried out with xylene and alcohol. Afterwards, the paraffin sections were digested with $20 \mu \mathrm{g} / \mathrm{ml}$ proteinase K (Sigma-Aldrich; Merck KGaA) at room temperature for $10 \mathrm{~min}$. Next, they were incubated with TUNEL assay solution (Promega Corporation) at $37^{\circ} \mathrm{C}$ for $90 \mathrm{~min}$, according to the manufacturer's protocol. Finally, representative images were taken from the hippocampal region in a total of four different sections per animal using a VS120 Virtual Slide Microscope (Olympus Corporation) at a magnification of $x 200$. Five randomly selected fields in each image were examined. The brown colored apoptotic cells were identified as 'TUNEL positive cells' and the percentage of the TUNEL positive cells were calculated.

Western blotting. A total of $100 \mathrm{mg}$ infarcted cerebral cortex tissue was cut into small pieces, and $1 \mathrm{ml}$ RIPA lysis buffer (Beyotime Institute of Biotechnology) and $1 \mathrm{ml}$ PMSF (final concentration, $1 \mathrm{mM}$; Beyotime Institute of Biotechnology) were added to the tissue, which was then homogenized untilfully lysed. After centrifugation at $30,000 \times \mathrm{g}$ for $3 \mathrm{~min}$ at $4^{\circ} \mathrm{C}$, the supernatant was collected. The protein concentration in supernatants was determined using a standard BCA protein assay kit (Beyotime Institute of Biotechnology), then $40 \mu \mathrm{g}$ protein in the supernatant was transferred to polyvinylidene difluoride (PVDF) membranes using Mini-Protean Tetra Electrophoresis System (Bio-Rad Laboratories, Inc.). Afterwards, PVDF membranes were blocked in 5\% skimmed milk at room temperature for $2 \mathrm{~h}$. They were incubated with primary antibodies against Caspase-3 (cat. no. sc-271759; 1:1,000; Santa Cruz Biotechnology, Inc.), Caspase-8 (cat. no. sc-5263; 1:1,000; Santa Cruz Biotechnology, Inc.), Caspase-9 (cat. no. sc-133109; 1:1,000; Santa Cruz Biotechnology, Inc.), Bax (sc-20067; 1:1,000; Santa Cruz Biotechnology, Inc.), Bcl-2 (cat. no. sc-56015; 1:1,000; Santa Cruz Biotechnology, Inc.), GFAP (cat. no. sc-33673; 1:1,000, Santa Cruz Biotechnology, Inc.) and AIF1 (cat. no. sc-32725; 1:1,000; Santa Cruz Biotechnology, Inc.) at $4{ }^{\circ} \mathrm{C}$ overnight. The next day, they were incubated with a horseradish peroxidase-conjugated secondary antibody (cat. no. G-21040; 1:10,000; Thermo Fisher Scientific, Inc.) at $37^{\circ} \mathrm{C}$ for $2 \mathrm{~h}$. The enhanced chemiluminescent substrate was applied to the blot membrane and incubated at room temperature for $5 \mathrm{~min}$. The blot membrane was then placed in a chemiluminescence imager (Bio-Rad Laboratories, Inc.) for image acquisition, and densitometry analysis was performed using ImageJ 1.43 software (National Institutes of Health).

Statistical analysis. Statistical analysis was performed using two-way analysis of variance method followed by Turkey post hoc test with SPSS software 17.0 (SPSS, Inc.). Data were represented as the mean \pm standard error of the mean. The number of experimental repeats was 12 . $\mathrm{P}<0.05$ was considered to indicate a statistically significant difference.

\section{Results}

Treatment with the ROCK inhibitor increases survival following MCAO. Survival checks were performed at 24 and $48 \mathrm{~h}$ and 7 days after MCAO surgery. As shown in Table I, the survival rates at 24 and $48 \mathrm{~h}$ and 7 days in Control and Y-27632 groups were 100\%, suggesting that Y-27632 alone did not affect overall survival. In the MCAO + Vehicle group, the survival rates at 24 and $48 \mathrm{~h}$ and 7 days were 60, 50 and $35 \%$, respectively, with only seven animals surviving until the seventh day after the MCAO surgery. In the MCAO + Y-27632 group, however, the survival rates at $24,48 \mathrm{~h}$ and 7 days were significantly higher at 90,80 and $50 \%$, respectively, with 10 rats surviving until the seventh day after the MCAO surgery.

$Y$-27632 reduces the MCAO-induced infarct volume in the brain. To assess the effect of ROCK inhibitor Y-27632 on the infarct caused by MCAO, the infarcted brain area was stained with TTC and compared between groups. As shown in Fig. 2, the infarct volume in the MCAO group was $31.5 \pm 3.6 \%$, $\mathrm{MCAO}+$ Vehicle group was $28.3 \pm 5.1 \%$, while the infarct volume in the MCAO + Y-27632 group was $18.2 \pm 3.3 \%$. There was no significant difference between MCAO group and $\mathrm{MCAO}+$ Vehicle group. The infarct size difference between $\mathrm{MCAO}+$ Vehicle and MCAO + Y-27632 group was statistically significant $(\mathrm{P}<0.05)$.

Y-27632 prevents neurological impairments caused by CIR. As shown in Fig. 3A, MCAO induced significant neurological deficits. The deficit score in the MCAO + Vehicle group was $3.1 \pm 0.23$ ( $\mathrm{P}<0.05$ compared to Control). There was no significant difference between MCAO group and MCAO + Vehicle group. However, the application of the ROCK inhibitor Y-27632 significantly decreased the neurological deficit score to $2.2 \pm 0.31$ ( $\mathrm{P}<0.05$ compared to $\mathrm{MCAO}+$ Vehicle $)$. The parameters evaluated with the water maze test are presented in Fig. 3B-F. The mean swimming speed (Fig. 3B) was not different between these four groups, suggesting that the motor ability of rats were not altered by MCAO or Y-27632. Following treatment with Y-27632, the escape latency and travel length during training days (Fig. 3C and D) were significantly decreased compared to MCAO + Vehicle $(\mathrm{P}<0.05)$. Rats in MCAO + Vehicle group on the test day (sixth day; Fig. 3E) crossed the hidden platform significantly less, while Y-27632 treated animals significantly increased the number of crosses $(\mathrm{P}<0.05$ compared to $\mathrm{MCAO}+$ Vehicle $)$. There was no significant difference between MCAO group and MCAO + Vehicle group. The time rats spent in target quadrant on the test day (sixth day; Fig. 3F) was significantly longer in the $\mathrm{MCAO}+\mathrm{Y}-27632$ group compared with the MCAO + Vehicle group $(\mathrm{P}<0.05)$. Y-27632 alone did not affect all these behavior test results ( $\mathrm{P}>0.05$ compared to Control).

ROCK inhibitor attenuates the histological changes caused by CIR. The histological changes to the hippocampus across 
Table I. Effect of Y-27632 on rat survival rate.

\begin{tabular}{|c|c|c|c|c|c|c|}
\hline & \multicolumn{6}{|c|}{ Survival } \\
\hline & \multicolumn{2}{|c|}{$24 \mathrm{~h}$} & \multicolumn{2}{|c|}{$48 \mathrm{~h}$} & \multicolumn{2}{|c|}{7 days } \\
\hline & $\mathrm{n}$ & $\%$ & $\mathrm{n}$ & $\%$ & $\mathrm{~N}$ & $\%$ \\
\hline Control & $12 / 12$ & 100 & $12 / 12$ & 100 & $12 / 12$ & 100 \\
\hline Y-27632 & $12 / 12$ & 100 & $12 / 12$ & 100 & $12 / 12$ & 100 \\
\hline MCAO+Vehicle & $12 / 20^{\mathrm{a}}$ & 60 & $10 / 20^{\mathrm{a}}$ & 50 & $7 / 20^{\mathrm{a}}$ & 35 \\
\hline MCAO+Y-27632 & $18 / 20^{\mathrm{b}}$ & 90 & $16 / 20^{\mathrm{b}}$ & 80 & $10 / 20^{\mathrm{b}}$ & 50 \\
\hline
\end{tabular}

$\mathrm{n}$, number of survivors/number of rats in the group. MCAO, middle cerebral artery occlusion. ${ }^{\mathrm{a}} \mathrm{P}<0.05$ compared to Control; ${ }^{\text {b }} \mathrm{P}<0.05$ compared to MCAO+Vehicle.

A

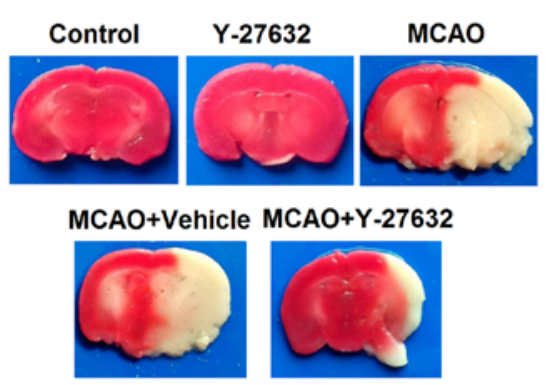

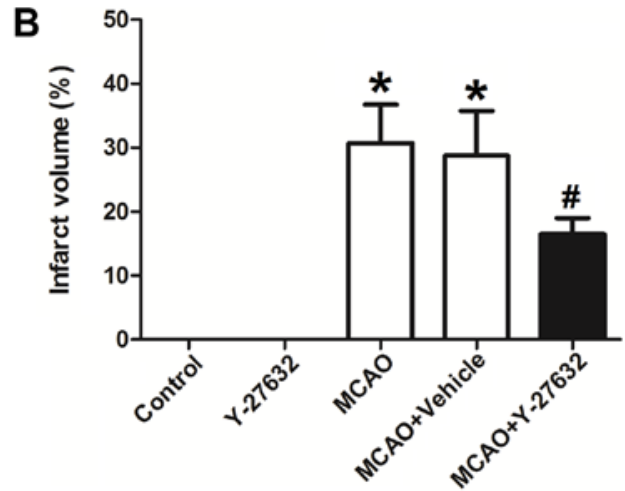

Figure 2. Influence of Y-27632 on the infarct volume. At $24 \mathrm{~h}$ after MCAO, rats were sacrificed, and the brains were collected for TTC staining. (A) Representative images of the infarct area. (B) Quantification of infarct volume of the Control, MCAO + Vehicle and MCAO + Y-27632 groups ( $\mathrm{n}=12$ per group). $\mathrm{P}<0.05$ compared to the Control group; ${ }^{~} \mathrm{P}<0.05$ compared to the $\mathrm{MCAO}+$ Vehicle group. MCAO, middle cerebral artery occlusion; TTC, 2,3,5-triphenyltetrazolium chloride.

the four groups are shown in Fig. 4. In the MCAO and $\mathrm{MCAO}+$ Vehicle groups, the brain tissue became less compact, the extracellular space was widened. In the MCAO + Y-27632 group, these observations were significantly less common.

Treatment with the ROCK inhibitor attenuates the oxidative injury and inflammation. The changes in oxidative injury indicators across groups are displayed in Fig. 5A-C. The levels of MDA and 3-NT (Fig. 5A and C) in the MCAO + Vehicle group were greatly increased compared to Control, and were significantly inhibited by co-treatment with Y-27632. The levels of 8-OHdG (Fig. 5B) was greatly increased compared to Control, but not changed by Y-27632. The changes in inflammatory marker expression across groups are shown in Fig. 5D-F. The levels of TNF- $\alpha$ and IL-1 $\beta$ (Fig. 5D and E) in the MCAO + Vehicle group were significantly increased compared to Control, and were inhibited by co-treatment with $\mathrm{Y}-27632(\mathrm{P}<0.05$ compared to MCAO + Vehicle). The IL-6 levels (Fig. 5F) were also increased compared to Control, but not significantly changed by Y-27632. Y-27632 alone did not affect oxidative injury or inflammation ( $\mathrm{P}>0.05$ compared to Control).

$Y$-27632 attenuates the expression of NSE in hippocampus tissue. The expression of NSE, a marker of CIR-induced damage, in the hippocampus of Control, Y-27632, MCAO
+ Vehicle and MCAO + Y-27632 groups is shown in Fig. 6. Representative images of IHC staining for NSE are shown in Fig. 6A, while Fig. 6B shows the quantification results of NSE expression. The results indicated that the expression of NSE was significantly increased following MCAO, but inhibited by the treatment with Y-27632 ( $\mathrm{P}<0.05$ compared to $\mathrm{MCAO}+$ Vehicle).

Y-27632 attenuates neuron apoptosis caused by CIR. To analyze the effect of ROCK inhibition on neuron apoptosis caused by CIR, the apoptosis rate in the hippocampus tissue and the protein levels of Caspase-3, Caspase- 8 and Caspase- 9 and the Bax/Bcl-2 ratio in the infarcted brain tissues were evaluated (Fig. 7). Representative images of the TUNEL assay are shown in Fig. 7A, while Fig. 7B indicates the quantification of apoptotic numbers in the hippocampus. The rate of apoptosis was greatly increased by MCAO, but reduced with $\mathrm{Y}-27632$ treatment $(\mathrm{P}<0.05$ compared to $\mathrm{MCAO}+$ Vehicle $)$. Y-27632 alone did not change the rate of apoptosis. As illustrated in Fig. 8, the protein levels of Caspase-3, Caspase-8 and Caspase-9 were also increased following MCAO, but reduced with Y-27632 treatment. Furthermore, Y-27632 significantly decreased the $\mathrm{Bax} / \mathrm{Bcl}-2$ ratio in infarcted brain tissues. Y-27632 alone did not affect neuron apoptosis $(\mathrm{P}>0.05$ compared to Control). 
A

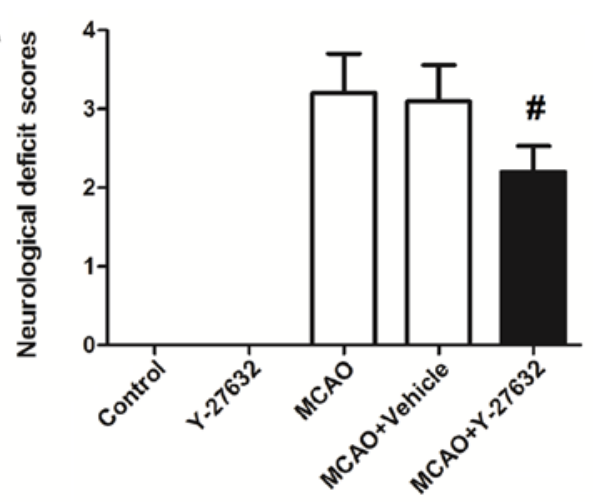

C

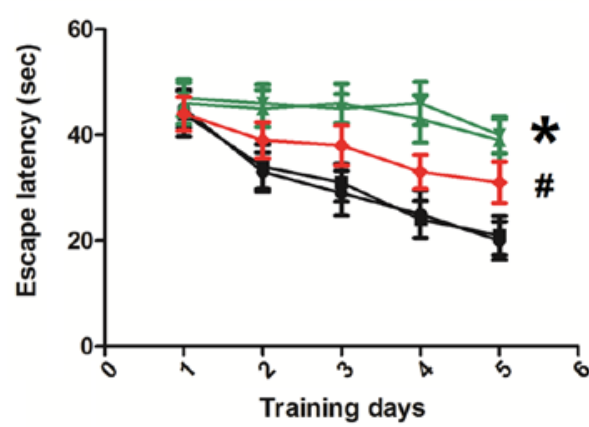

E

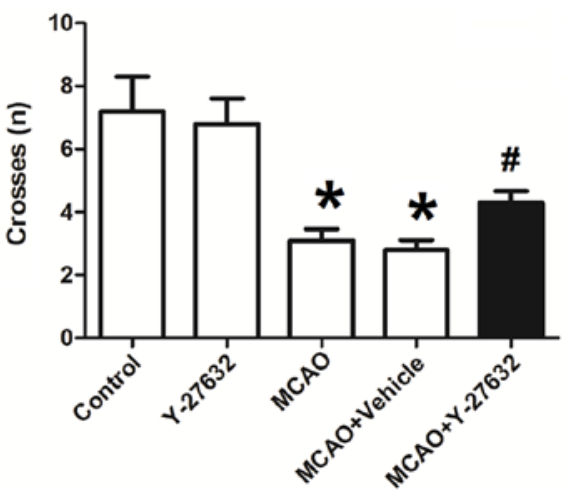

B

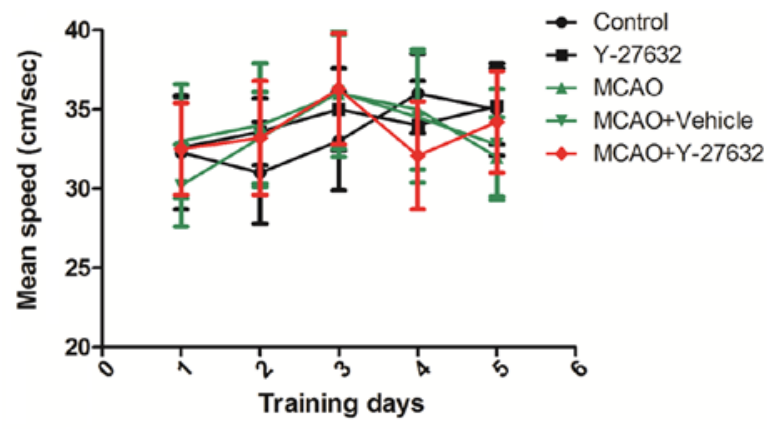

D

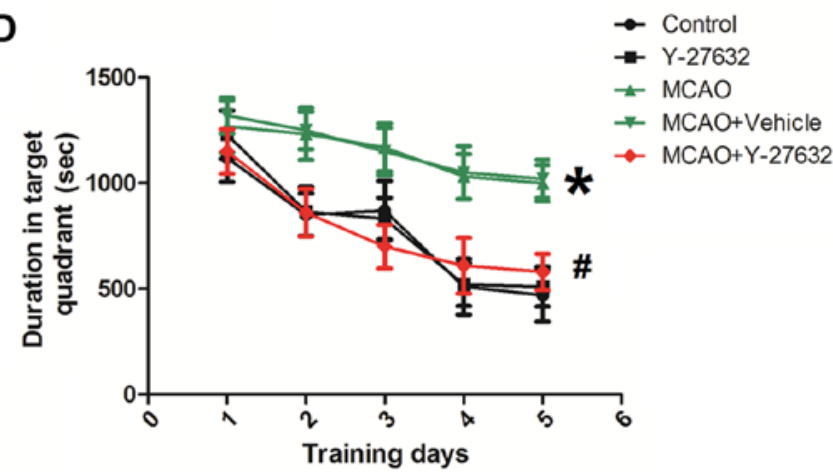

$\mathbf{F}$

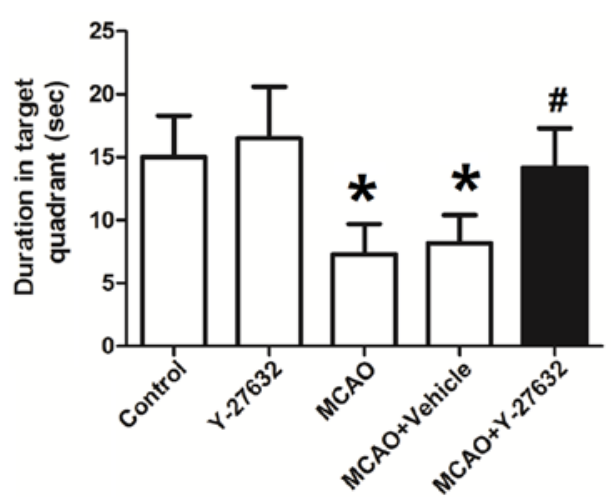

Figure 3. Influence of Y-27632 on the neurological impairment caused by cerebral ischemia-reperfusion injury. (A) Results of neurological deficit tests. (B-F) Results of the several parameters evaluated using the eater maze test. Each column represents the mean \pm standard error of the mean of each parameter for animals in each group ( $\mathrm{n}=12$ per group). ${ }^{*} \mathrm{P}<0.05$ compared to the Control group; ${ }^{\#} \mathrm{P}<0.05$ compared to the MCAO $+\mathrm{Vehicle}$ group. $\mathrm{MCAO}$, middle cerebral artery occlusion.

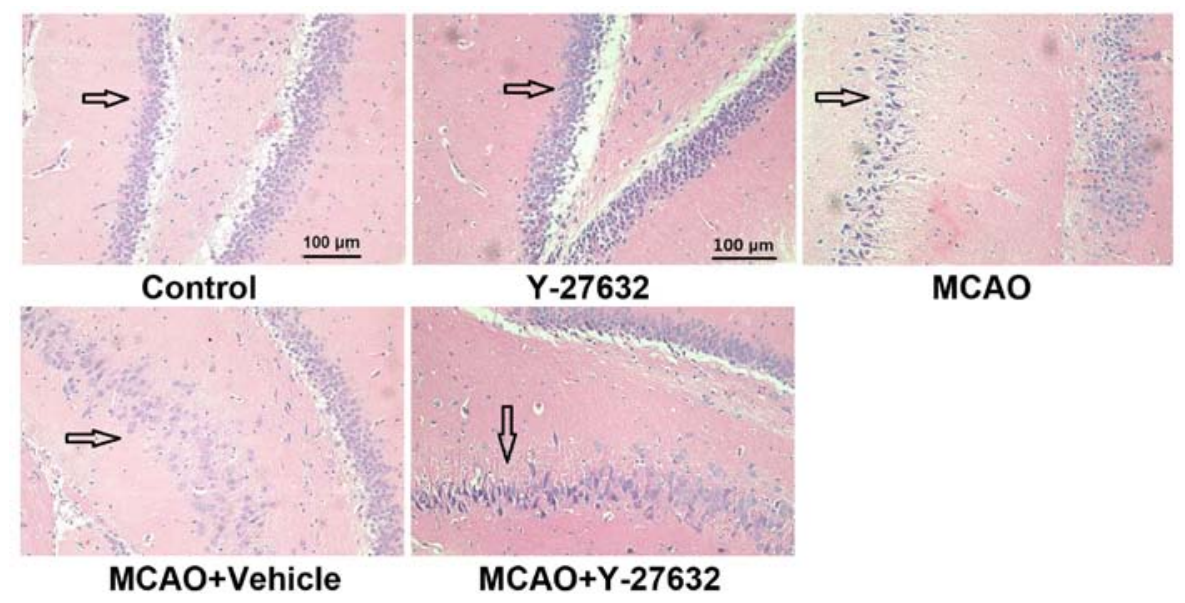

Figure 4. Influence of Y-27632 on the histological changes caused by cerebral ischemia-reperfusion injury. The black arrows are pointing at the whole group of cells in the hippocampal tissues. In the MCAO and MCAO + Vehicle groups, the brain tissue became less compact, the extracellular space was widened. In the MCAO + Y-27632 group, these observations were significantly less common ( $\mathrm{n}=12$ per group). MCAO, middle cerebral artery occlusion. 
A
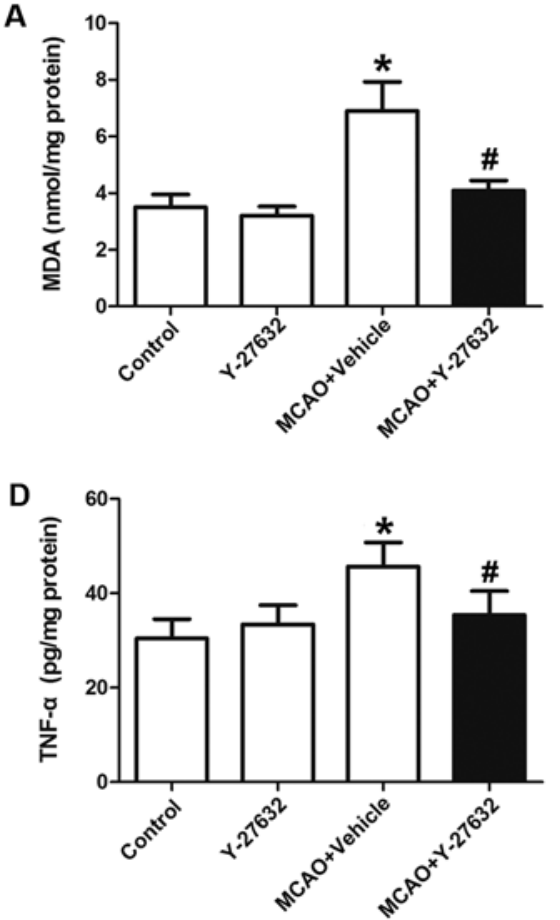

B

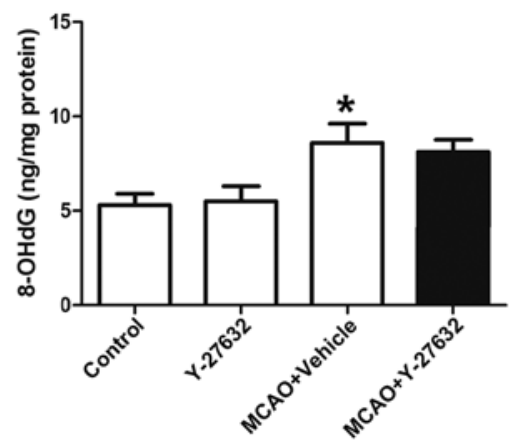

E

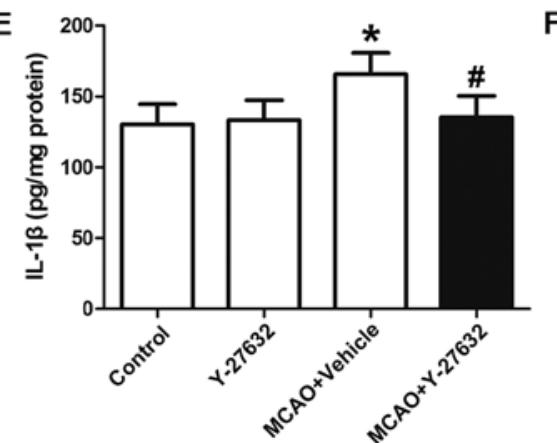

C
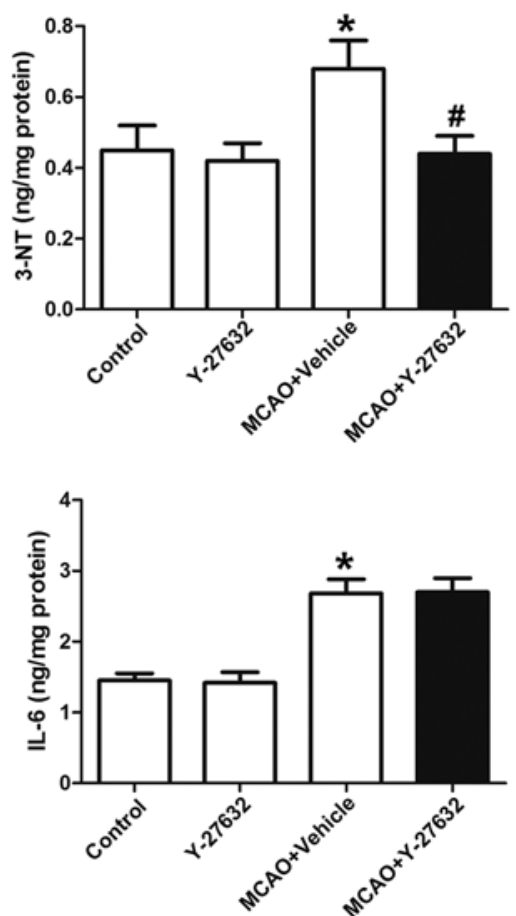

Figure 5. Influence of Y-27632 on the oxidative injury and inflammation markers following cerebral ischemia-reperfusion injury. (A-C) Levels of MDA, 8-OHdG and 3-NT. (D-F) Levels of TNF- $\alpha$, IL-1 $\beta$ and IL-6. Each column represents the mean \pm standard error of the mean of each parameter for animals in each group ( $\mathrm{n}=12$ per group). ${ }^{*} \mathrm{P}<0.05$ compared to the Control group; ${ }^{*} \mathrm{P}<0.05$ compared to the $\mathrm{MCAO}+$ Vehicle group. MDA, malondialdehyde; 8 -OHdG, 8-hydroxy-2'-deoxyguanosine; 3-NT, 3-nitrotyrosine; MCAO, middle cerebral artery occlusion.

A

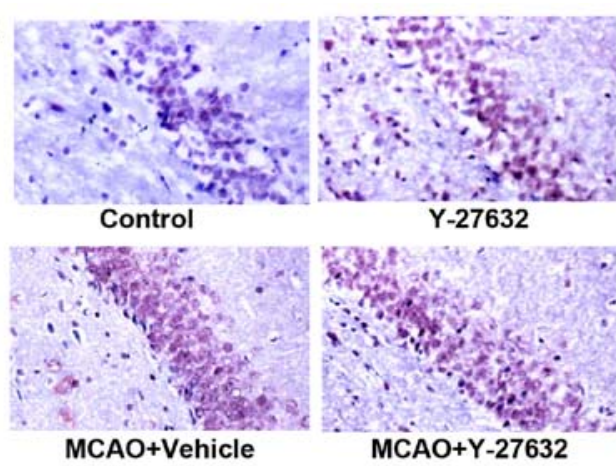

B

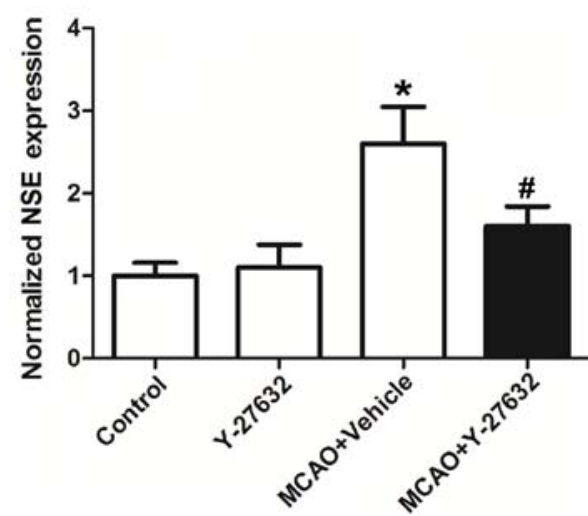

Figure 6. Influence of Y-27632 on the expression of NSE in the hippocampus. To investigate the effects of Y-27632, the expression of NSE, a marker of cerebral damage, was evaluated in the hippocampus using immunohistochemical staining. (A) Representative images of immunohistochemical staining (magnification $\mathrm{x} 400$ ). (B) Quantification of NSE expression in brain slices ( $\mathrm{n}=12$ per group). ${ }^{*} \mathrm{P}<0.05$ compared to the Control group; ${ }^{*} \mathrm{P}<0.05$ compared to the $\mathrm{MCAO}+$ Vehicle group. NSE, neuron specific enolase; MCAO, middle cerebral artery occlusion.

$Y$-27632 increases the levels of GFAP and AIF1 following $M C A O$. To explore the effect of ROCK inhibition on astrocytes and microglia function, the levels of GFAP and AIF1 were evaluated in infarcted brain tissues by western blotting. Representative images of western blotting for GFAP and the quantification of its expression are shown in Fig. 9A, while Fig. 9B presents representative images of AIF1 western blot analysisAIF1 and respective quantification of its expression. The results demonstrated that the protein levels of GFAP and AIF1 were both increased by MCAO, and the treatment of Y-27632 further increased these protein levels to a higher level following MCAO. Y-27632 alone did not affect GFAP and AIF1 protein levels ( $\mathrm{P}>0.05$ compared to Control).

\section{Discussion}

The present study investigated the effects of the ROCK inhibitor Y-27632 on CIR injury and explored its underlying mechanism of action. It demonstrated that ROCK inhibition by Y-27632 significantly improved the cognitive ability that was impaired following CIR damage. Y-27632 also reduced the expression of oxidative injury and inflammation markers in the brain and attenuated the histological changes in the hippocampus caused by the injury. The level of neuronal apoptosis in CIR-injured rats was also significantly decreased by Y-27632 treatment. Lastly, the protein levels of GFAP and AIF1 were further increased following Y-27632 exposure and 

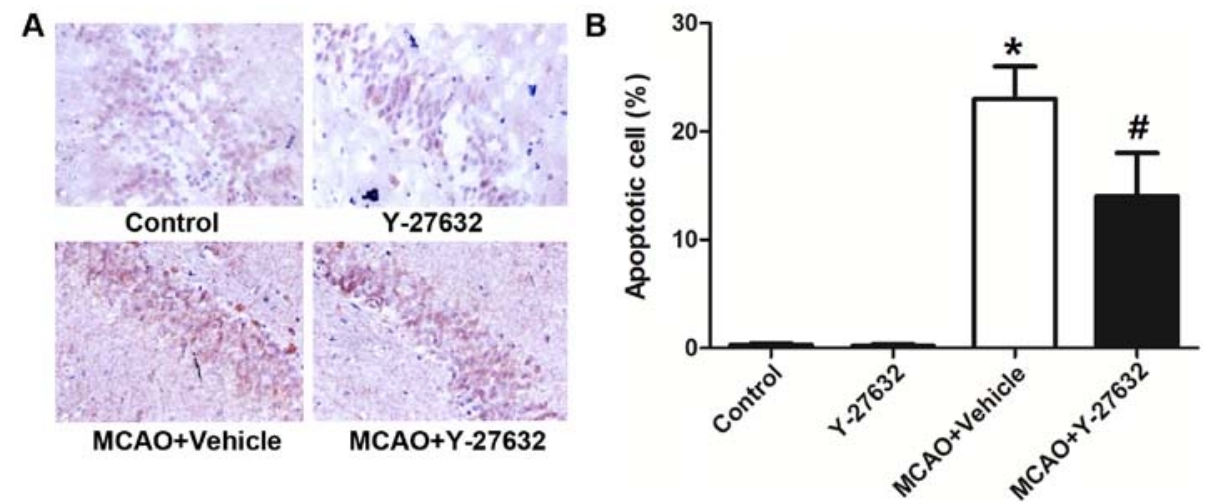

Figure 7. Influence of Y-27632 on the neuron apoptosis caused by CIR. To measure the influence of Y-27632 on neuron apoptosis caused by CIR, the hippocampal tissues from Control, Y-27632, MCAO + Vehicle, MCAO + Y-27632 groups were stained using the TUNEL method. (A) Representative images of TUNEL staining (magnification $\mathrm{x} 400$ ). (B) Percentage of TUNEL-positive cells in each treatment group ( $\mathrm{n}=12$ per group). ${ }^{*} \mathrm{P}<0.05$ compared to the Control group; ${ }^{\# P}<0.05$ compared to the MCAO + Vehicle group. CIR, cerebral ischemia-reperfusion injury; MCAO, middle cerebral artery occlusion; TUNEL, terminal deoxynucleotidyl transferase dUTP nick end labeling.

A

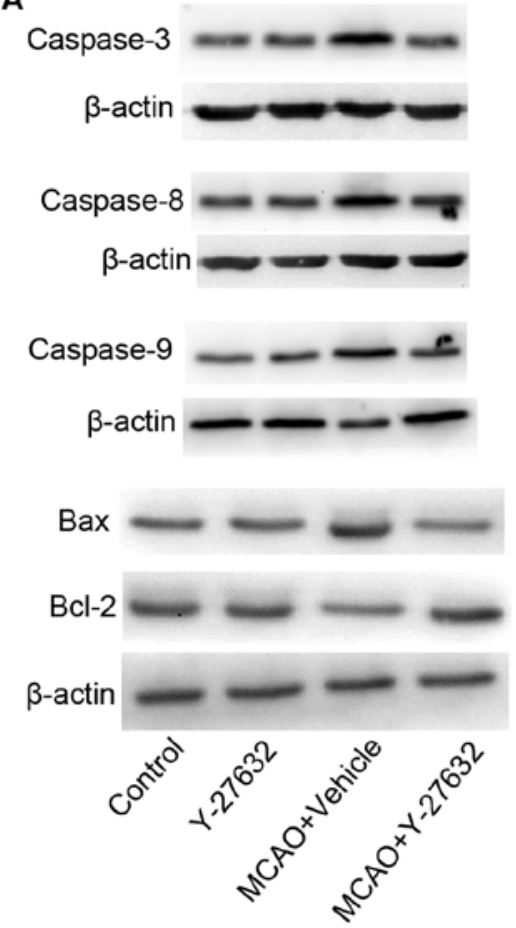

B

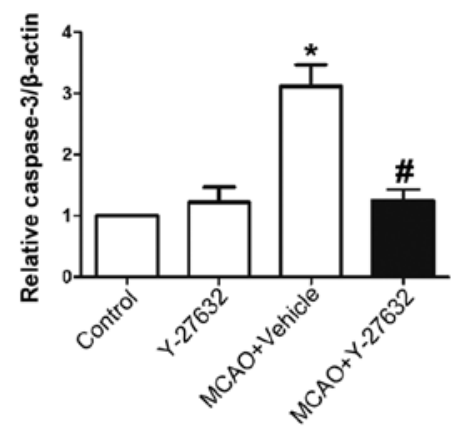

D

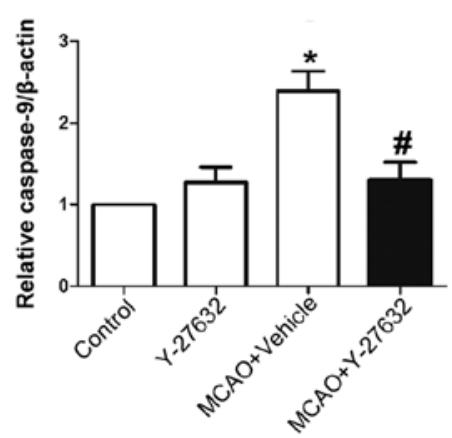

C

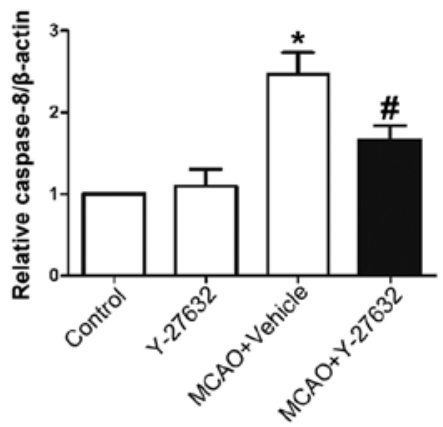

E

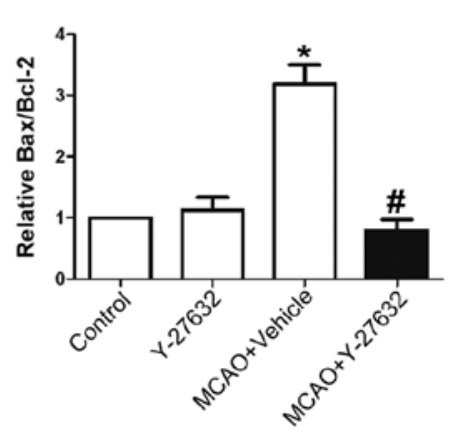

Figure 8. Influence of Y-27632 on the expression of apoptotic proteins. (A) Representative bands of Caspase-3, Caspase-8, Caspase-9, Bax and Bcl-2 in each group. (B-E) Relative levels of Caspase-3, Caspase-8, Caspase-9 Bax/Bcl-2 ratio. Each column represents the mean \pm standard error of the mean of expression values for each treatment group ( $\mathrm{n}=12$ per group). ${ }^{*} \mathrm{P}<0.05$ compared to the Control group; ${ }^{*} \mathrm{P}<0.05$ compared to the MCAO + Vehicle group. MCAO, middle cerebral artery occlusion.

Y-27632 significantly increased the survival rates at $24,48 \mathrm{~h}$ and 7 days after CIR.

ROCK is an important kinase involved in cell mitosis, adhesion, cytoskeleton regulation, muscle cell contraction, tumor cell infiltration/migration and other cell functions (16). ROCK participates in the signaling pathway that regulates the presence of its respective receptors in the cytoplasm and is also involved in the regulation of the cytoskeletal structure (17). In recent years, ROCK has been increasingly studied in context of IR injury (18-20). The pretreatment of heart tissue with fusadil may prevent the endothelial dysfunction and significantly reduce the degree of myocardial infarction (21). In the early stages of reperfusion, upregulation of Rho expression in the ischemic myocardium activates ROCK (22). To evaluate the potential beneficial effect of ROCK inhibition on CIR, rats with MCAO were treated with the ROCK inhibitor Y-27632 and their survival rates and neurological performance were examined. The survival rate of animals treated with MCAO and Vehicle at 24, $48 \mathrm{~h}$ and 7 days was 60,50 and 35\%, respectively. In contrast, MCAO animals treated with Y-27632 group exhibited significantly higher survival rates $(90,80$ and $50 \%$ at 24 and $48 \mathrm{~h}$ and 7 days respectively). Furthermore, Y-27632 significantly decreased the neurological deficit score and enhanced the performance of rats in the water maze tests 
A
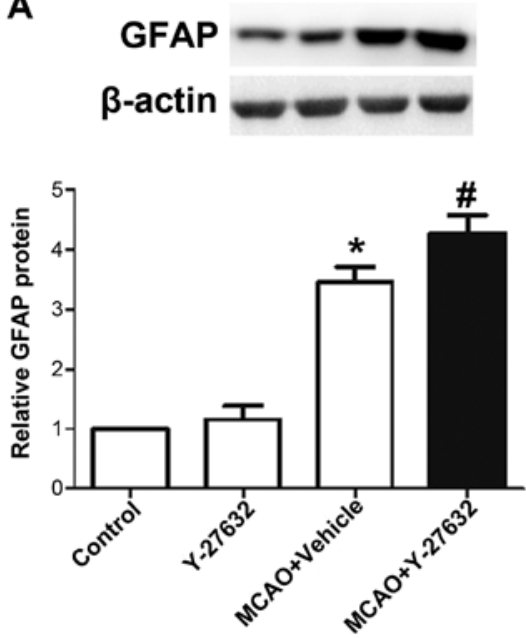

B
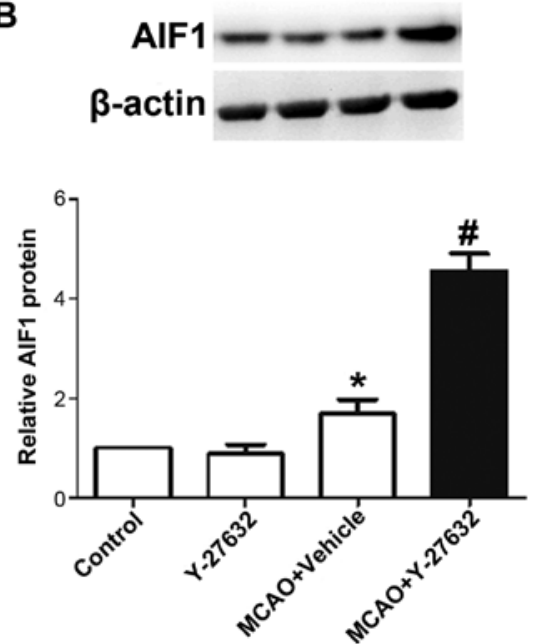

Figure 9. Influence of Y-27632 on the levels of GFAP and AIF1 following cerebral ischemia-reperfusion injury. (A) GFAP and (B) AIF1 expression results and quantification. Each column represents the mean \pm standard error of the mean of expression values for each treatment group ( $\mathrm{n}=12$ per group). $\mathrm{P}<0.05$ compared to the Control group; ${ }^{\prime} \mathrm{P}<0.05$ compared to the $\mathrm{MCAO}+$ Vehicle group. GFAP, glial fibrillary acidic protein; AIF1, allograft inflammatory factor 1 ; MCAO, middle cerebral artery occlusion.

compared to MCAO + Vehicle animals. These results indicated that ROCK inhibition may effectively prevent death and the neurological impairment caused by CIR.

Different factors, including ischemia, hypoxia, hyperglycemia and infection, may activate tyrosine kinases and $\mathrm{G}$ protein-coupled receptors, which in turn activate RhoA. Activation of the RhoA/ROCK signaling pathway may have detrimental effects during IR. Firstly, activation of the RhoA/ROCK signaling pathway in the vascular endothelium is known to inhibit P13K/AKT activation, decrease nitric oxide synthase 3 phosphorylation and nitric oxide production, which may reduce regional cerebral blood flow following CIR (23). Secondly, activation of RhoA/ROCK signaling pathway may increase blood flow viscosity. The exact mechanism remains unclear, but is hypothesized to be associated with erythrocyte deformability (24). Thirdly, activation of the RhoA/ROCK signaling pathway promotes the expression of serpin family $\mathrm{E}$ member 2 in vascular endothelial cells and worsens intracapillary circulation (25). Phosphorylation of myosin light-chain in vascular endothelial cells can lead to a decrease in tight junctions between cells and increased endothelial cell permeability (26). Lastly, activation of the RhoA/ROCK signaling pathway may increase the infiltration of inflammatory (neutrophils) into ischemic tissue, aggravating the inflammatory response during IR, and reducing the integrity of the blood-brain barrier (27). The mechanism underlying fusadil's protection against cerebral infarction includes increased local cerebral blood flow by vasodilation and reduced inflammatory response (28). To evaluate the effect of ROCK inhibition on inflammatory response induced following CIR injury, rats with MCAO were treated with Y-27632 and the levels of pro-inflammatory factors TNF- $\alpha$, IL-1 $\beta$ and IL- 6 in the infarcted brain tissue were examined (29-30). The levels of TNF- $\alpha$ and IL-1 $\beta$ in MCAO + Vehicle group were significantly increased compared to Control, and treatment with Y-27632 significantly inhibited their increase, suggesting the involvement of ROCK signaling pathway in the inflammatory response induced by CIR. MCAO also induced significant histological changes in the hippocampus, and these were significantly attenuated following treatment with the ROCK inhibitor. Overall, these results indicated that Y-27632 may have directly attenuated the inflammatory response and reduced hippocampal injury induced by CIR.

Free oxygen radicals are an important product in IR injuries. These may increase the activity of arginase in endothelial cells through Protein kinase C-activated RhoA/ROCK signaling pathway, reduce the production of nitric oxide, increase superoxide production and disturb normal endothelial function (31). Moreover, the RhoA/ROCK signaling pathway has been shown to reduce the production of free oxygen radicals following cerebral infarction (32). As the present study showed, the levels of MDA and 3-NT in MCAO + Vehicle group were greatly increased compared to Control, and treatment of Y-27632 prevented this increase. This result indicated that the protective effect of Y-27632 against CIR injury may also be associated with reduced oxidative stress.

When the central nervous system is injured, astrocytes will transform from a resting state to an activated state (and are hence termed 'reactive astrocytes'). Reactive gliosis results in the hypertrophy of glial cells and in the proliferation of microglia (33). The response of astrocytes to injury is thought to promote the protection of the nervous system and the isolation of the injury area from the surrounding healthy tissue. After the injury, activated astrocytes and microglia may increase the secretion of neurotrophic factors (including nerve growth factor, neurotrophin-3, brain-derived neurotrophic factor), which can maintain the survival of neurons and accelerate the growth of neurites (34). The release of these factors also allows activated glial cells to provide neurons with 'scaffolds' that help restore disrupted neural connections. In the infarcted area, the expression level of a variety of growth factors secreted by astrocytes increases significantly, which provides direct nutritional support for neurons and oligodendrocytes and limits secondary damage (35). Hypoxia, inflammatory response and trauma can promote activated astrocytes to secrete vascular endothelial growth factor (VEGF). VEGF may also protect neurons and promote angiogenesis and formation of new 
blood vessels, which is beneficial for the repair of damaged areas (36). By eliminating glial cells or by selectively blocking a signal pathway, a study has demonstrated that the key to barrier remodeling is to activate proliferation and migration of astrocytes (37). Therefore, to explore the potential role of astrocytes and microglia in the Y-27632-mediate protection against CIR injury, the protein levels of GFAP (a marker of astrocyte) and AIF1 (a marker of microglia) in the infarcted area were evaluated. The results demonstrated that the protein levels of GFAP and AIF1 were greatly increased by CIR, as expected considering the damage caused to the neuronal tissue. However, the treatment of Y-27632 further increased these protein levels. These results confirmed that astrocytes and microglia would are more active in response to CIR. It also revealed that Y-27632 may stimulate their response and exert protective effects via activation of both astrocytes and microglia. Previous studies have found that the inhibition of the ROCK pathway attenuated methylmercury-induced astrocyte death (38). It was also revealed that ROCK inhibitors Y-27632 and fasudil promote microglial migration in the spinal cord via the ERK signaling pathway (39). Consistent with these studies, the present study confirmed that activation of astrocytes and microglia may be a possible mechanism by which ROCK inhibition may protect brain tissue against CIR injury.

However, the present study had some limitations. Firstly, multiple mechanisms may be associated with Y-27632-induced protection against CIR. The present study focused on the activation of astrocytes and microglia, but this may not be the only mechanism. Secondly, this is a primary animal study on the protective effect of Y-27632 in CIR injury. Many factors should be investigated when considering its clinical application against CIR, including, but not limited to, its effects on human beings, toxicity and dosage. Thirdly, further mechanism should be investigated in future studies, such as how Y-27632 regulates the activation of astrocytes and microglia or the interaction between ROCK and astrocytes and microglia. Finally, the study would be more perfect if the formation of the glial scar was shown by immunohistochemical examination, but because of the limitation of time and the technology, we are unable to perform it.

In conclusion, the present study confirmed that Y-27632, a ROCK inhibitor, effectively increased the survival rate and behavioral performance of rats with CIR damage. It also decreased the oxidative stress, cerebral inflammation, neuron apoptosis and hippocampal damage in animals with MCAO. The activated proliferation of astrocytes and microglia may be responsible for this beneficial effect.

\section{Acknowledgements}

Not applicable.

\section{Funding}

No funding was received.

\section{Availability of data and materials}

The datasets used and/or analyzed during the current study are available from the corresponding author on reasonable request.

\section{Authors' contributions}

LL conceived and designed the study and revised the manuscript; BL performed the experiments and performed the statistical analysis. All authors read and approved the manuscript.

\section{Ethics approval and consent to participate}

The present study was approved by the Ethics Committee of Tianjin Baodi District People's Hospital.

\section{Patient consent for publication}

Not applicable.

\section{Competing interests}

The authors declare that they have no competing interests.

\section{References}

1. Iadecola C and Anrather J: Stroke research at a crossroad: Asking the brain for directions. Nat Neurosci 14: 1363-1368, 2011.

2. Breuer L, Knott M, Struffert T, Kloska S, Kurka N, Schwab S, Dörfler A, Köhrmann $M$ and Engelhorn T: Limited versus whole-brain perfusion for the indication of thrombolysis in the extended time window of acute cerebral ischemia. J Stroke Cerebrovasc Dis 24: 2491-2496, 2015.

3. Xiong XY, Liu L and Yang QW: Refocusing neuroprotection in cerebral reperfusion era: New challenges and strategies. Front Neurol 9: 249, 2018.

4. Panieri E, Gogvadze V, Norberg E, Venkatesh R, Orrenius S and Zhivotovsky B: Reactive oxygen species generated in different compartments induce cell death, survival, or senescence. Free Radic Biol Med 57: 176-187, 2013.

5. Xue J, Zhang X, Zhang C, Kang N, Liu X, Yu J, Zhang N, Wang H, Zhang L, Chen R, et al: Protective effect of Naoxintong against cerebral ischemia reperfusion injury in mice. J Ethnopharmacol 182: 181-189, 2016.

6. Julian L and Olson MF: Rho-associated coiled-coil containing kinases (ROCK): Structure, regulation, and functions. Small GTPases 5: e29846, 2014.

7. Schmandke A, Schmandke A and Strittmatter SM: ROCK and Rho: Biochemistry and neuronal functions of Rho-associated protein kinases. Neuroscientist 13: 454-469, 2007.

8. Gong $\mathrm{H}$ and Yang CY: Morphological and hydrodynamic correlations with increasing outflow facility by rho-kinase inhibitor Y-27632. J Ocul Pharmacol Ther 30: 143-153, 2014.

9. Oda T, Kume T, Izumi Y, Ishihara K, Sugmimoto H and Akaike A: $\mathrm{Na}^{+} / \mathrm{Ca}^{2+}$ exchanger inhibitors inhibit neurite outgrowth in PC12 cells. J Pharmacol Sci 116: 128-131, 2011.

10. Wang J, Li H, Yao Y, Ren Y, Lin J, Hu J, Zheng M, Song X, Zhao T, Chen YY, et al: $\beta$-elemene enhances GAP-43 expression and neurite outgrowth by inhibiting RhoA kinase activation in rats with spinal cord injury. Neuroscience 383: 12-21, 2018.

11. Pang Y, Chai CR, Gao K, Jia XH, Kong JG, Chen XQ, Vatcher G, Chen JG and $\mathrm{Yu} A C$ : Ischemia preconditioning protects astrocytes from ischemic injury through 14-3-3 $\gamma$. J Neurosci Res 93: 1507-1518, 2015.

12. Zhao JJ, Hu JX, Lu DX, Ji CX, Qi Y, Liu XY, Sun FY, Huang F, $\mathrm{Xu} \mathrm{P}$ and Chen XH: Soluble cpg15 from astrocytes ameliorates neurite outgrowth recovery of hippocampal neurons after mouse cerebral ischemia. J Neurosci 37: 1628-1647, 2017.

13. Yang $\mathrm{C}$, Zhang $\mathrm{X}$, Fan $\mathrm{H}$ and Liu Y: Curcumin upregulates transcription factor Nrf2, HO-1 expression and protects rat brains against focal ischemia. Brain Res 28: 133-141, 2009.

14. Jia D, Deng Y, Gao J, Liu X, Chu J and Shu Y: Neuroprotective effect of Panax notoginseng plysaccharides against focal cerebral ischemia reperfusion injury in rats. Int J Biol Macromol 63: 177-180, 2014. 
15. Ahmadi M, Rajaei Z, Hadjzadeh MA, Nemati H and Hosseini M: Crocin improves spatial learning and memory deficits in the Morris water maze via attenuating cortical oxidative damage in diabetic rats. Neurosci Lett 642: 1-6, 2017.

16. Mueller BK, Mack H and Teusch N: Rho kinase, a promising drug target for neurological disorders. Nat Rev Drug Discov 4: 387-398, 2005

17. Tanaka T, Nishimura D, Wu RC, Amano M, Iso T, Kedes L, Nishida H, Kaibuchi K and Hamamori Y: Nuclear Rho kinase, ROCK 2, targets p300 acetyltransferase. J Biol Chem 281: 15320-15329, 2006.

18. Wen JY, Gao SS, Chen FL, Chen S, Wang M and Chen ZW: Role of CSE-produced $\mathrm{H} 2 \mathrm{~S}$ on cerebrovascular relaxation via RhoA-ROCK inhibition and cerebral ischemia-reperfusion injury in Mice. ACS Chem Neurosci 10: 1565-1574, 2019.

19. Sun XQ, Chen S, Wang LF and Chen ZW: Total flavones of Rhododendron simsii Planch flower protect isolated rat heart from ischaemia-reperfusion injury and its mechanism of UTR-RhoA-ROCK pathway inhibition. J Pharm Pharmacol 70: 1713-1722, 2018

20. Chen F, Liu Z, Peng W, Gao Z, Ouyang H, Yan T, Ding S, Cai Z, Zhao B, Mao L and Cao Z: Activation of EphA4 induced by EphrinA1 exacerbates disruption of the blood-brain barrier following cerebral ischemia-reperfusion via the Rho/ROCK signaling pathway. Exp Ther Med 16: 2651-2658, 2018.

21. Yada T, Shimokawa H, Hiramatsu O, Kajita T, Shigeto F, Tanaka E, Shinozaki Y, Mori H, Kiyooka T, Katsura M, et al: Beneficial effect of hydroxyfasudil, a specific Rho-kinase inhibitor, on ischemia/reperfusion injury in canine coronary microcirculation in vivo. J Am Coll Cardiol 45: 599-607, 2005.

22. Hamid SA, Bower HS and Baxter GF: Rho kinase activation plays a major role as a mediator of irreversible injury in reperfused myocardium. Am J Physiol Heart Circ Physiol 292: H2598-H2606, 2007.

23. Shin HK, Salomone S, Potts EM, Lee SW, Millican E, Noma K, Huang PL, Boas DA, Liao JK, Moskowitz MA and Ayata C: Rho-kinase inhibition acutely augments blood flow in focal cerebral ischemia via endothelial mechanisms. J Cereb Blood Flow Metab 27: 998-1009, 2007.

24. Tiftik RN, Baskurt OK, Kul S and Buyukafsar K: The functional significance of the rho/rho-kinase pathway in human erythrocytes. Turk J Haematol 31: 168-174, 2014.

25. Iwasaki H, Okamoto $R$, Kato S, Konishi K, Mizutani $H$, Yamada N, Isaka N, Nakano T and Ito M: High glucose induces plasminogen activator inhibitor-1 expression through Rho/Rho-kinase-mediated NF-kappaB activation in bovine aortic endothelial cells. Atherosclerosis 196: 22-28, 2008.

26. Shen L, Black ED, Witkowski ED, Lencer WI, Guerriero V, Schneeberger EE and Turner JR: Myosin light chain phosphorylation regulates barrier function by remodeling tight junction structure. J Cell Sci 119: 2095-2106, 2006.
27. Satoh S, Utsunomiya T, Tsurui K, Kobayashi T, Ikegaki I, Sasaki Y and Asano T: Pharmacological profile of hydroxy fasudil as a selective rho kinase inhibitor on ischemic brain damage. Life Sci 69: 1441-1453, 2001

28. Rikitake Y, Kim HH, Huang Z, Seto M, Yano K, Asano T, Moskowitz MA and Liao JK: Inhibition of Rho kinase (ROCK) leads to increased cerebral blood flow and stroke protection. Stroke 36: 2251-2257, 2005

29. Lu Z, Cheng D, Yin J, Wu R, Zhang G, Zhao Q, Wang N, Wang F and Liang M: Antithrombin III protects against contrast-induced nephropathy. EBioMedicine 17: 101-107, 2017.

30. Yin J, Wang F, Kong Y, Wu R, Zhang G, Wang N, Wang L, Lu Z and Liang M: Antithrombin III prevents progression of chronic kidney disease following experimental ischaemic-reperfusion injury. J Cell Mol Med 21: 3506-3514, 2017

31. Chandra S, Romero MJ, Shatanawi A,Alkilany AM,Caldwell RB and Caldwell RW: Oxidative species increase arginase activity in endothelial cells through the RhoA/Rho kinase pathway. $\mathrm{Br}$ J Pharmacol 165: 506-519, 2012.

32. Kahles T, Luedike P, Endres M, Galla HJ, Steinmetz H, Busse R, Neumann-Haefelin T and Brandes RP: NADPH oxidase plays a central role in blood-brain barrier damage in experimental stroke. Stroke 38: 3000-3006, 2007.

33. Gao Z, Zhu Q, Zhang Y, Zhao Y, Cai L, Shields CB and Cai J: Reciprocal modulation between microglia and astrocyte in reactive gliosis following the CNS injury. Mol Neurobiol 48: 690-701, 2013.

34. Cragnolini AB, Montenegro G, Friedman WJ and Masco DH: Brain-region specific responses of astrocytes to an in vitro injury and neurotrophins. Mol Cell Neurosci 88: 240-248, 2018.

35. Williams A,Piaton G and Lubetzki C: Astrocytes-friends or foes in multiple sclerosis? Glia 55: 1300-1312, 2007.

36. Guo D, Murdoch CE, Xu H, Shi H, Duan DD, Ahmed A and $\mathrm{Gu}$ Y: Vascular endothelial growth factor signaling requires glycine to promote angiogenesis. Sci Rep 7: 14749, 2017

37. Okada S, Nakamura M, Katoh H, Miyao T, Shimazaki T, Ishii K, Yamane J, Yoshimura A, Iwamoto Y, Toyama Y and Okano H: Conditional ablation of Stat 3 or Socs 3 discloses a dual role for reactive astrocytes after spinal cord injury. Nat Med 12: 829-834, 2006.

38. Dos Santos AA, Lopez-Granero C, Farina M, Rocha JBT, Bowman $\mathrm{AB}$ and Aschner M: Oxidative stress, caspase-3 activation and cleavage of ROCK-1 play an essential role in $\mathrm{MeHg}$-induced cell death in primary astroglial cells. Food Chem Toxicol 113: 328-336, 2018

39. Fu PC, Tang RH, Yu ZY, Xie MJ, Wang W and Luo X: The Rho-associated kinase inhibitors Y27632 and fasudil promote microglial migration in the spinal cord via the ERK signaling pathway. Neural Regen Res 13: 677-683, 2018. 